\title{
HANKEL OPERATORS WITH WEIGHTED LIPSCHITZ SYMBOLS IN THE UNIT BALL
}

\author{
BENOÎT F. SEHBA and EDGAR TCHOUNDJA
}

\begin{abstract}
We characterize symbols of bounded Hankel operators between some Hardy-Orlicz spaces and usual Hardy spaces in the unit ball of $\mathrm{C}^{n}$.
\end{abstract}

\section{Introduction}

Let $\mathrm{B}^{n}=\left\{z \in \mathrm{C}^{n}:|z|<1\right\}$ be the unit ball of $\mathrm{C}^{n}(n \geq 1)$. We denote by $d v$ the normalized Lebesgue measure on $\mathrm{B}^{n}$ and $d \sigma$ the normalized measure on $\mathrm{S}^{n}=\partial \mathrm{B}^{n}$ the boundary of $\mathrm{B}^{n}$. By $\mathscr{H}\left(\mathrm{B}^{n}\right)$, we denote the space of holomorphic functions on $\mathrm{B}^{n}$.

For $z=\left(z_{1}, \ldots, z_{n}\right)$ and $w=\left(w_{1}, \ldots, w_{n}\right)$ in $\mathrm{C}^{n}$, we let

$$
\langle z, w\rangle=z_{1} \overline{w_{1}}+\cdots+z_{n} \overline{w_{n}}
$$

so that $|z|^{2}=\langle z, z\rangle=\left|z_{1}\right|^{2}+\cdots+\left|z_{n}\right|^{2}$.

For $\Phi$ a continuous and nondecreasing function from $[0, \infty)$ onto itself with the property that $\Phi$ is of strictly positive lower type, that is, we can find $\epsilon>0$ and $C>0$ such that, for $s>0$ and $0<t \leq 1$,

$$
\Phi(s t) \leq C t^{\epsilon} \Phi(s),
$$

we denote by $\mathscr{H}^{\Phi}\left(\mathrm{B}^{n}\right)$ the Hardy-Orlicz space consisting of holomorphic function $f$ in the unit ball $\mathrm{B}^{n}$ such that if the functions $f_{r}$ are defined by $f_{r}(z)=f(r z)$ then

$$
\|f\|_{\mathscr{C}^{\Phi}}:=\sup _{r<1}\left\|f_{r}\right\|_{L^{\Phi}}^{\mathrm{lux}}<\infty
$$

where

$$
\|f\|_{L^{\Phi}}^{\operatorname{lux}}:=\inf \left\{\lambda>0: \int_{\varsigma^{n}} \Phi\left(\frac{|f(\xi)|}{\lambda}\right) d \sigma(\xi) \leq 1\right\}
$$

is the Luxemboug (quasi)-norm of $f$ in the Orlicz space $L^{\Phi}\left(\mathbf{S}^{n}\right)$. 
For $0<p<\infty$, when $\Phi(t)=t^{p}$, the above space corresponds to the usual Hardy space $\mathscr{H}^{p}\left(\mathrm{~B}^{n}\right)$, that is the space of all $f \in \mathscr{H}\left(\mathrm{B}^{n}\right)$ such that

$$
\|f\|_{p}^{p}:=\sup _{0<r<1} \int_{S^{n}}|f(r \xi)|^{p} d \sigma(\xi)<\infty .
$$

We denote by $\mathscr{C}^{\infty}\left(\mathrm{B}^{n}\right)$, the space of bounded holomorphic functions in $\mathrm{B}^{n}$.

For any $\xi \in \mathbf{S}^{n}$ and $\delta>0$, let

$$
B_{\delta}(\xi)=\left\{w \in \mathrm{S}^{n}:|1-\langle w, \xi\rangle|<\delta\right\},
$$

and

$$
Q_{\delta}(\xi)=\left\{z \in \mathrm{B}^{n}:|1-\langle z, \xi\rangle|<\delta\right\} .
$$

These are the higher dimension analogues of Carleson regions. We call a weight $\varrho$, any continuous increasing function from $[0, \infty)$ onto itself, which is of upper type $\alpha$ on $[0,1]$, that is,

$$
\varrho(s t) \leq s^{\alpha} \varrho(t)
$$

for $s>1$, with $s t \leq 1$. Given a weight $\varrho$, we define the space $B M O(\varrho)$ as the subspace of $L^{2}\left(\mathbf{S}^{n}\right)$ consisting of those $f \in L^{2}\left(\mathbf{S}^{n}\right)$ such that

$$
\sup _{B} \inf _{R \in \mathscr{P}_{N}(B)} \frac{1}{(\varrho(\sigma(B)))^{2} \sigma(B)} \int_{B}|f-R|^{2} d \sigma=C<\infty,
$$

where, for $B=B_{\delta}\left(\xi_{0}\right)$, the space $\mathscr{P}_{N}(B)$ is the space of polynomials of order $\leq N$ in the $(2 n-1)$ last coordinates related to an orthonormal basis whose first element is $\xi_{0}$ and second element $\operatorname{Im} \xi_{0}$. Here $N$ is taken larger than $2 n \alpha-1$. We note $\|f\|_{B M O(\varrho)}:=\|f\|_{2}+C$, where $C$ is given in the definition (1.3) of $B M O(\varrho)$. The above definition does not depend on the choice of $N$ (see [1], [5]). The space $B M O A(\varrho)$ is then the space of function $f \in \mathscr{H}^{2}\left(\mathrm{~B}^{n}\right)$ such that

$$
\sup _{r<1}\left\|f_{r}\right\|_{B M O(\rho)}<\infty .
$$

Clearly, $B M O A(\varrho)$ coincides with the space of holomorphic functions in $\mathscr{H}^{2}\left(\mathrm{~B}^{n}\right)$ such that their boundary values lie in $B M O(\varrho)$. When $\varrho=1, B M O A(\varrho)$ is the usual space of holomorphic functions with bounded mean oscillation $B M O A$; it is the dual space of the Hardy space $\mathscr{H}^{1}\left(\mathrm{~B}^{n}\right)$.

When $\Phi$ is given by $\Phi_{p}(t)=\frac{t^{p}}{\log (e+t)^{p}}, 0<p \leq 1$, any function $f$ in $\mathscr{H}^{\Phi_{p}}\left(\mathrm{~B}^{n}\right)$ can be weakly factorized in terms of functions in $\mathscr{H}^{p}\left(\mathrm{~B}^{n}\right)$ and functions in BMOA. More precisely, we have the following result obtained in [1], [6]. 

hold.

TheOREM 1.1. Let $0<p \leq 1$, and $\Phi_{p}(t)=\frac{t^{p}}{\log (e+t)^{p}}$. Then the following

(1) The product of two functions, one in $\mathscr{H}^{p}\left(\mathrm{~B}^{n}\right)$ and the other one in $B M O A$, is in $\mathscr{H}^{\Phi_{p}}\left(\mathrm{~B}^{n}\right)$. Moreover, $\|f g\|_{\mathscr{H}^{\Phi_{p}}} \lesssim\|f\|_{\mathscr{H}^{p}}\|g\|_{B M O A}$.

(2) Any function $f \in \mathscr{H}^{\Phi_{p}}\left(\mathrm{~B}^{n}\right)$ can be represented (weakly factorized) as $f=\sum_{j} f_{j} g_{j}$ with $\sum_{j}\left\|f_{j}\right\|_{\mathscr{C} P}\left\|g_{j}\right\|_{B M O A} \leq C\|f\|_{\mathscr{C}^{\Phi}}$.

For simplicity, we will be using the notation $\mathscr{H}_{s}\left(\mathrm{~B}^{n}\right)$ for $\mathscr{H}^{\Phi}\left(\mathrm{B}^{n}\right)$ when $\Phi(s)=\frac{t^{s}}{\log (e+t)^{s}}, 0<s \leq 1$.

The orthogonal projection of $L^{2}\left(\partial \mathrm{B}^{n}\right)$ onto $\mathscr{H}^{2}\left(\mathrm{~B}^{n}\right)$ is called the Szegó projection and denoted $P$. It is given by

$$
P(f)(z)=\int_{\partial \mathrm{B}^{n}} S(z, \xi) f(\xi) d \sigma(\xi),
$$

where $S(z, \xi)=\frac{1}{(1-\langle z, \xi\rangle)^{n}}$ is the Szegó kernel on $\partial \mathrm{B}^{n}$. We denote as well by $P$ its extension to $L^{1}\left(\partial \mathrm{B}^{n}\right)$.

For $b \in \mathscr{H}^{2}\left(\mathrm{~B}^{n}\right)$, the small Hankel operator with symbol $b$ is defined for $f$ a bounded holomorphic function by $h_{b}(f):=P(b \bar{f})$.

We are interested here to the boundedness of the small Hankel operators $h_{b}$ from $\mathscr{H}^{p}\left(\mathrm{~B}^{n}\right)$ or $\mathscr{H}_{s}\left(\mathrm{~B}^{n}\right)$ to $\mathscr{H}^{q}\left(\mathrm{~B}^{n}\right)$, with $0<q<\infty$ and $0<p, s<1$. The continuity of this operator between Hardy spaces in one dimension has been considered in [10] and completely solved in [15]. Also in one dimension, A. Bonami and S. Madan gave in [4] a characterization of symbols of bounded Hankel operators between Hardy-Orlicz spaces in terms of the so called "balayage" of Carleson measures. In higher dimension, it is well-known that $h_{b}$ extends as a bounded operator on $\mathscr{H}^{p}\left(\mathrm{~B}^{n}\right)$ for $p>1$ if and only if $b$ is in $B M O A$ (see [7]). In [1], A. Bonami and S. Grellier using weak factorization results were able to characterize symbols of bounded Hankel operators from the space $\mathscr{H}_{p}\left(\mathrm{~B}^{n}\right), 0<p \leq 1$ to $\mathscr{H}^{1}\left(\mathrm{~B}^{n}\right)$. More direct method has been used in [2] for the case of Hankel operators from $\mathscr{H}^{1}\left(\mathrm{~B}^{n}\right)$ to itself. The two last works have been extended in [6] to the case of Hankel operators between two Hardy-Orlicz spaces under some conditions on the Orlicz functions involved.

To be more precise at this point, let us denote by $\wp_{\epsilon}$ the set of concave growth functions of lower type $\epsilon$, which are $\mathscr{C}^{1}$ functions and satisfy $\Phi^{\prime}(t) \simeq \frac{\Phi(t)}{t}$. For a weight $\varrho$, we call $\Psi_{\varrho}$ the following function first introduced in [8] and defined for $t>0$ by

$$
\Psi_{\varrho}(t):=\int_{t}^{\infty} \min \left(\frac{1}{s^{2}}, \frac{\varrho(s)}{s}\right) d s .
$$

A. Bonami and the first author have proved the following. 
THEOREM 1.2 ([6]). Let $\Phi_{1}$ and $\Phi_{2}$ in $\wp_{\epsilon}$, and $\varrho_{i}(t)=\frac{1}{t \Phi_{i}^{-1}(1 / t)}$. Assume that $b \in B M O A(\varrho)$, with

$$
\varrho:=\frac{\varrho_{1}}{\psi_{\varrho_{2}}}
$$

Then the Hankel operator $h_{b}$ extends into a continuous operator from $\mathscr{H}^{\Phi_{1}}\left(\mathrm{~B}^{n}\right)$ to $\mathscr{H}^{\Phi_{2}}\left(\mathrm{~B}^{n}\right)$. Moreover the condition is necessary under one of the following conditions: $\Phi_{2}$ is of lower type $\frac{n}{n+1}$, or satisfies the Dini condition $\int_{1}^{\infty} \frac{\Phi_{2}(t)}{t^{2}} d t<\infty$

Restricting to the spaces considered in this paper, the above result tells us that the ranges of symbols of bounded Hankel operators, $h_{b}$, from $\mathscr{H}^{p}\left(\mathrm{~B}^{n}\right)$ and $\mathscr{H}_{p}\left(\mathrm{~B}^{n}\right)$ to $\mathscr{H}^{q}\left(\mathrm{~B}^{n}\right)$ and $\mathscr{H}_{q}\left(\mathrm{~B}^{n}\right)$ for $0<p, q \leq 1$, are some appropriate weighted $B M O A$ spaces. Their proof follows from a weak factorization result of Hardy-Orlicz spaces and a duality argument between these spaces and weighted BMOA spaces. We see that because of the hypothesis of concavity, the image spaces $\mathscr{H}^{q}\left(\mathrm{~B}^{n}\right)$ are restricted to the range $0<q \leq 1$ and their result does not provide any answer for example in the case $h_{b}: \mathscr{H}_{p}\left(\mathrm{~B}^{n}\right) \rightarrow \mathscr{H}^{q}\left(\mathrm{~B}^{n}\right)$ when $q>1$.

The aims of this paper is to give a simple and explicit characterization of the symbols of bounded Hankel operators from $\mathscr{H}^{p}\left(\mathrm{~B}^{n}\right)$ or $\mathscr{H}_{s}\left(\mathrm{~B}^{n}\right)$ to $\mathscr{H}^{q}\left(\mathrm{~B}^{n}\right)$, with $1 \leq q<\infty$ and $0<p, s<1$. This is a particular case of the boundedness of the Hankel operator $h_{b}$ from $\mathscr{H}^{\Phi_{1}}\left(\mathrm{~B}^{n}\right)$ to $\mathscr{H}^{\Phi_{2}}\left(\mathrm{~B}^{n}\right)$ with $\Phi_{1}$ concave and $\Phi_{2}$ convex. We restrict ourselves to this case which can be handled using less technical methods than [1] and [6], the general case being considered in a forthcoming paper. To deal with the case $h_{b}: \mathscr{H}^{p}\left(\mathrm{~B}^{n}\right) \rightarrow \mathscr{H}^{q}\left(\mathrm{~B}^{n}\right)$, we only need to adapt the method of [3] to our situation while in the case of $h_{b}: \mathscr{H}_{p}\left(\mathrm{~B}^{n}\right) \rightarrow \mathscr{H}^{q}\left(\mathrm{~B}^{n}\right)$, we combine this method with the weak factorization result provided by Theorem 1.1. This approach allows us to prove that the range of symbols of bounded Hankel operators between these spaces is a weighted (logarithmic) Lipschitz space. When $0<p, q \leq 1$, the characterization in Theorem 1.2 can be also interpreted in terms of Lipschitz spaces. Another proof can be given in this case by following for example [11, Chapter IV].

Finally, all over the text, $C$ will be a constant not necessary the same at each occurrence. We will also use the notation $C_{k}$ to express the fact that the constant depends on the underlined parameter. Given two positive quantities $A$ and $B$, the notation $A \lesssim B$ means that $A \leq C B$ for some positive constant $C$. When $A \lesssim B$ and $B \lesssim A$, we write $A \simeq B$. 


\section{Preliminaries}

We introduce here some useful lemmas. For this, we recall that given an analytic function $f$ on $\mathrm{B}^{n}$, the radial derivative $R f$ of $f$ is defined by

$$
R f(z)=\sum_{j=1}^{n} z_{j} \frac{\partial f}{\partial z_{j}}(z) .
$$

For $\alpha \geq 0$, we denote by $\Gamma_{\alpha}\left(\mathrm{B}^{n}\right)$ the space of holomorphic functions $f$ for which there exist and integer $k>\alpha$ and a positive constant $C$ such that

$$
\left|R^{k} f(z)\right| \leq C\left(1-|z|^{2}\right)^{\alpha-k} .
$$

Remark that for $\alpha=0$, the class $\Gamma_{\alpha}\left(\mathrm{B}^{n}\right)$ coincides with the usual Bloch class. For $\alpha>0$, it coincides with the class of Lipschitz functions of order $\alpha$. For $t \in \mathrm{R}$, we also define the logarithmic Lipschitz space $L \Gamma_{\alpha}^{t}\left(\mathrm{~B}^{n}\right)$ as the space of holomorphic function $f$ in $\mathrm{B}^{n}$ such that, for some $k>\alpha$

$$
\left|R^{k} f(z)\right| \leq C\left(1-|z|^{2}\right)^{\alpha-k}\left(\log \frac{1}{1-|z|^{2}}\right)^{-t} .
$$

When $t=1$, we write $L \Gamma_{\alpha}^{1}\left(\mathrm{~B}^{n}\right)=L \Gamma_{\alpha}\left(\mathrm{B}^{n}\right)$.

The spaces, $\Gamma_{\alpha}\left(\mathrm{B}^{n}\right)$ and $L \Gamma_{\alpha}^{t}\left(\mathrm{~B}^{n}\right)$, become Banach spaces under the following norms

$$
\begin{aligned}
\|f\|_{\Gamma_{\alpha}\left(\mathrm{B}^{n}\right)} & =|f(0)|+\sup _{z \in \mathrm{B}^{n}}\left|R^{k} f(z)\right|\left(1-|z|^{2}\right)^{k-\alpha} \\
\|f\|_{L \Gamma_{\alpha}^{t}\left(\mathrm{~B}^{n}\right)} & =|f(0)|+\sup _{z \in \mathrm{B}^{n}}\left|R^{k} f(z)\right|\left(1-|z|^{2}\right)^{k-\alpha}\left(\log \frac{1}{1-|z|^{2}}\right)^{t},
\end{aligned}
$$

where $k$ is a fixed integer such that $k>\alpha$. It is well known that these spaces are the same for various values of $k$.

We will need the following differential operator of order $k$ :

$$
M_{k}=[(n+k-1) I+R] \cdots[(n+1) I+R][n I+R]
$$

where $I$ is the identity operator, $k \in \mathrm{N}$. We prove the following useful lemma.

Lemma 2.1. Let $f, g$ be holomorphic polynomials on $\mathrm{B}^{n}$. Then the following equality holds

$$
\int_{\mathrm{S}^{n}} f(\xi) \bar{g}(\xi) d \sigma(\xi)=C_{k} \int_{\mathrm{B}^{n}} f(z) \overline{M_{k} g(z)}\left(1-|z|^{2}\right)^{k-1} d v(z) .
$$


Proof. We only need to check (2.1) for $f(z)=z^{m}$ and $g(z)=z^{l}$, where $m=\left(m_{1}, \ldots, m_{n}\right)$ and $l=\left(l_{1}, \ldots, l_{n}\right)$ are multi-indexes of nonnegative integers. We recall that $z^{m}=z_{1}^{m_{1}} \cdots z_{n}^{m_{n}}$ and write $|m|=m_{1}+\cdots+m_{n}$. Let us write

$$
I_{m, l}=\int_{\varsigma^{n}} f(\xi) \bar{g}(\xi) d \sigma(\xi)=\int_{\varsigma^{n}} \xi^{m} \overline{\xi^{l}} d \sigma(\xi)
$$

and

$$
\begin{aligned}
J_{m, l} & =\int_{\mathrm{B}^{n}} f(z) \overline{M_{k} g(z)}\left(1-|z|^{2}\right)^{k-1} d v(z) \\
& =\frac{(n+|l|+k-1) !}{(n+|l|-1) !} \int_{\mathrm{B}^{n}} z^{m} \overline{z^{l}}\left(1-|z|^{2}\right)^{k-1} d v(z) .
\end{aligned}
$$

We have for $m \neq l, I_{m, l}=0=J_{m, l}$ (see [16]) and for $m=l$, it follows from [16, Lemma 1.11] that

$$
I_{m, m}=\int_{S^{n}}\left|\xi^{m}\right|^{2} d \sigma(\xi)=\frac{(n-1) ! m !}{(n+|m|-1) !}=\frac{(n-1) ! m !}{\Gamma(n+|m|)}
$$

and

$$
\begin{aligned}
J_{m, m} & =\frac{(n+|m|+k-1) !}{(n+|m|-1) !} \int_{\mathrm{B}^{n}}\left|z^{m}\right|^{2}\left(1-|z|^{2}\right)^{k-1} d \nu(z) \\
& =\frac{n ! \Gamma(k)}{\Gamma(n+k)} \frac{m !(n+|m|+k-1) ! \Gamma(n+k)}{(n+|m|-1) ! \Gamma(n+|m|+k)} \\
& =\frac{n ! m ! \Gamma(k)}{\Gamma(n+|m|)}=n \Gamma(k) I_{m, m} .
\end{aligned}
$$

The proof is complete.

In particular, for any holomorphic polynomial $f$, we have

$$
\begin{aligned}
h_{b}(f)(z): & =P(b \bar{f})(z) \\
& =C_{k} \int_{\mathrm{B}^{n}} S(z, w) \bar{f}(w) M_{k} b(w)\left(1-|w|^{2}\right)^{k-1} d v(w) .
\end{aligned}
$$

As a consequence, one obtain

Lemma 2.2. Let $f$ and $g$ be two holomorphic polynomials. Then

$$
\begin{aligned}
\left\langle h_{b} f, g\right\rangle & :=\lim _{r \rightarrow 1} \int_{\mathrm{S}^{n}} h_{b}(f)(r \xi) \overline{g(\xi)} d \sigma(\xi) \\
& =C_{k} \int_{\mathrm{B}^{n}} \overline{f(z) g(z)} M_{k} b(z)\left(1-|z|^{2}\right)^{k-1} d \nu(z) .
\end{aligned}
$$


We recall the following lemma.

Lemma 2.3 (Lemma 2.2 of [3]). Let $\left(a_{j}\right)$ be a sequence of positive numbers, and let $L_{k}$ be the differential operator of order $k$ defined by

$$
L_{k}:=\left(a_{0} I+R\right)\left(a_{1} I+R\right) \cdots\left(a_{k-1} I+R\right) .
$$

Then $f$ belongs to $\Gamma_{\alpha}\left(\mathrm{B}^{n}\right)$ if and only there exist an integer $k>\alpha$ and $a$ positive constant $C$ such that

$$
\left|L_{k} f(z)\right| \leq C\left(1-|z|^{2}\right)^{\alpha-k} .
$$

As remarked in [3], the equivalence in the above lemma also holds if we multiply the right hand side of the last inequality by a logarithmic term. That is, for fixed $t \in \mathbf{R}$

$$
\left|R^{k} f(z)\right| \leq C\left(1-|z|^{2}\right)^{\alpha-k}\left|\log \left(1-|z|^{2}\right)\right|^{t}
$$

if and only if

$$
\left|L_{k} f(z)\right| \leq C\left(1-|z|^{2}\right)^{\alpha-k}\left|\log \left(1-|z|^{2}\right)\right|^{t} .
$$

A simple consequence of this lemma is that

$$
\begin{aligned}
\|f\|_{\Gamma_{\alpha}\left(\mathrm{B}^{n}\right)} & \simeq \sup _{z \in \mathrm{B}^{n}}\left|M_{k} f(z)\right|\left(1-|z|^{2}\right)^{k-\alpha} \\
\|f\|_{L \Gamma_{\alpha}^{t}\left(\mathrm{~B}^{n}\right)} & \simeq \sup _{z \in \mathrm{B}^{n}}\left|M_{k} f(z)\right|\left(1-|z|^{2}\right)^{k-\alpha}\left(\log \frac{1}{1-|z|^{2}}\right)^{t} .
\end{aligned}
$$

The next lemma is proved in [16, Theorem 4.48].

Lemma 2.4. For fixed $0<p<q<\infty, a=\frac{1}{p}-\frac{1}{q}$, there is a constant $C_{p, q}>0$ so that, for $f \in \mathscr{H}^{p}\left(\mathrm{~B}^{n}\right)$,

$$
\left(\int_{\mathrm{B}^{n}}|f(z)|^{q}\left(1-|z|^{2}\right)^{n q a-1} d \nu(z)\right)^{1 / q} \leq C_{p, q}\|f\|_{\mathscr{C}^{p}}
$$

If we take $q=1$ in the above lemma, we obtain the following useful inequality: let $0<p<1$, for every $f \in \mathscr{H}^{p}\left(\mathrm{~B}^{n}\right)$,

$$
\int_{\mathrm{B}^{n}}|f(z)|\left(1-|z|^{2}\right)^{n\left(\frac{1}{p}-1\right)-1} d v(z) \leq C\|f\|_{\mathscr{H}^{p}} .
$$

Let us observe the following. 
Lemma 2.5. Let $0<p<1$. Then for any $f \in \mathscr{H}_{p}\left(\mathrm{~B}^{n}\right)$,

$$
\int_{\mathrm{B}^{n}}|f(z)|\left(1-|z|^{2}\right)^{n\left(\frac{1}{p}-1\right)-1}\left(\log \frac{1}{1-|z|^{2}}\right)^{-1} d v(z) \leq C\|f\|_{\mathscr{H}_{p}} .
$$

Proof. Recall that any $f \in \mathscr{H}_{p}\left(\mathrm{~B}^{n}\right)$ admits a representation of the form $f=\sum_{j} f_{j} g_{j}$ with $f_{j} \in \mathscr{H}^{p}\left(\mathrm{~B}^{n}\right), g_{j} \in B M O A$ and $\sum_{j}\left\|f_{j}\right\|_{\mathscr{P} p}\left\|g_{j}\right\|_{B M O A} \leq$ $C\|f\|_{\mathscr{H}_{p}}$. Also, for $g \in B M O A$, the following holds

$$
|g(z)| \leq C\left(\log \frac{1}{1-|z|^{2}}\right)\|g\|_{B M O A} .
$$

Thus, combining this with (2.5), we obtain

$$
\begin{aligned}
I & :=\int_{\mathrm{B}^{n}}|f(z)|\left(1-|z|^{2}\right)^{n\left(\frac{1}{p}-1\right)-1}\left(\log \frac{1}{1-|z|^{2}}\right)^{-1} d v(z) \\
& \leq \sum_{j} \int_{\mathrm{B}^{n}}\left|f_{j}(z) \| g_{j}(z)\right|\left(1-|z|^{2}\right)^{n\left(\frac{1}{p}-1\right)-1}\left(\log \frac{1}{1-|z|^{2}}\right)^{-1} d v(z) \\
& \lesssim \sum_{j}\left\|g_{j}\right\|_{B M O A} \int_{\mathrm{B}^{n}}\left|f_{j}(z)\right|\left(1-|z|^{2}\right)^{n\left(\frac{1}{p}-1\right)-1} d v(z) \\
& \lesssim \sum_{j}\left\|f_{j}\right\|_{\mathscr{C}^{p}}\left\|g_{j}\right\|_{B M O A} \\
& \leq C\|f\|_{\mathscr{H}_{p}} .
\end{aligned}
$$

Recall now that $B M O A$ is the dual space of $\mathscr{C}^{1}\left(\mathrm{~B}^{n}\right)$ under the following pairing

$$
\langle f, g\rangle:=\lim _{r \rightarrow 1} \int_{S^{n}} f(r \xi) \bar{g}(\xi) d \sigma(\xi),
$$

$f \in \mathscr{H}^{1}\left(\mathrm{~B}^{n}\right)$ and $g \in B M O A$ (see [16, p. 172]).

In general for $\Phi \in \wp_{\epsilon}$, the dual space of $\mathscr{H}^{\Phi}\left(\mathrm{B}^{n}\right)$ can be identified with $B M O A(\varrho)$, under the pairing (2.7), whenever $\varrho(t)=\frac{1}{t \Phi^{-1}(1 / t)}$ (see [9]).

Let us do the following remark.

Remark 2.6. Let $\alpha=n\left(\frac{1}{p}-1\right), 0<p<1$. We observe that for $\varrho_{1}(t)=t^{\frac{1}{p}-1}$ and $\varrho_{2}(t)=\frac{t^{\frac{1}{p}-1}}{\log \frac{e}{t}}$ the spaces $\Gamma_{\alpha}\left(\mathrm{B}^{n}\right)$ and $L \Gamma_{\alpha}\left(\mathrm{B}^{n}\right)$ coincide with $B M O A\left(\varrho_{1}\right)$ and $B M O A\left(\varrho_{2}\right)$ respectively. Let us give a proof of this for $\varrho_{2}$ as this is well-known for $\varrho_{1}$ (see [9]). 
Proof. It is not hard to see using for example Lemma 2.1 and inequality (2.6), that any $f \in L \Gamma_{\alpha}\left(\mathrm{B}^{n}\right)$ defines an element of the dual space $\left(\mathscr{H}_{p}\left(\mathrm{~B}^{n}\right)\right)^{*}$ of $\mathscr{H}_{p}\left(\mathrm{~B}^{n}\right)$. Thus, $f \in B M O A\left(\varrho_{2}\right)=\left(\mathscr{H}_{p}\left(\mathrm{~B}^{n}\right)\right)^{*}$.

Conversely, let us suppose $f \in B M O A\left(\varrho_{2}\right)$ and prove that for all $z \in \mathrm{B}^{n}$,

$$
\left|R^{k} f(z)\right| \leq C\left(1-|z|^{2}\right)^{\alpha-k}\left(\log \frac{1}{1-|z|^{2}}\right)^{-1}
$$

for some integer $k>\alpha$. For this we first recall that if $\mu$ is the measure defined by $d \mu(z)=|R f(z)|^{2}\left(1-|z|^{2}\right) d \nu(z)$, then there is a constant $C>0$ such that for all $\delta \in(0,1)$ and $\xi \in \mathrm{S}^{n}$,

$$
\mu\left(Q_{\delta}(\xi)\right) \leq C \delta^{n+2 \alpha}\left(\log \frac{e}{\delta^{n}}\right)^{-2}
$$

(see [14]). Using reproducing formula for Bergman spaces [16], and the definition of radial derivative, we can find an absolute constant $C$ so that for some large $N$, some $\epsilon$ small enough and for some $k>\alpha$, and for all $z \in \mathrm{B}^{n}$

$$
\begin{aligned}
& \left|R^{k} f(z)\right| \\
& \quad \lesssim \int_{\mathrm{B}^{n}} \frac{|R f(w)|\left(1-|w|^{2}\right)^{N}}{|1-\langle z, w\rangle|^{n+N+k}} d v(w) \\
& \quad \lesssim\left(\int_{\mathrm{B}^{n}} \frac{|R f(w)|^{2}\left(1-|w|^{2}\right) d v(w)}{|1-\langle z, w\rangle|^{n+2 k-\epsilon}}\right)^{1 / 2}\left(\int_{\mathrm{B}^{n}} \frac{\left(1-|w|^{2}\right)^{2 N-1} d v(w)}{|1-\langle z, w\rangle|^{n+2 N+\epsilon}}\right)^{1 / 2} \\
& \quad \lesssim C\left(1-|z|^{2}\right)^{-\epsilon / 2}\left(\int_{\mathrm{B}^{n}} \frac{1}{|1-\langle z, w\rangle|^{n+2 k-\epsilon}} d \mu(w)\right)^{1 / 2} .
\end{aligned}
$$

Now standard arguments (see for example [13]), using (2.8) give:

$$
\int_{\mathrm{B}^{n}} \frac{1}{|1-\langle z, w\rangle|^{n+2 k-\epsilon}} d \mu(w) \leq C \frac{1}{\left(1-|z|^{2}\right)^{2 k-2 \alpha-\epsilon}}\left(\log \frac{1}{1-|z|^{2}}\right)^{-2} .
$$

Hence, for all $z \in \mathrm{B}^{n}$,

$$
\left|R^{k} f(z)\right| \leq C\left(1-|z|^{2}\right)^{\alpha-k}\left(\log \frac{1}{1-|z|^{2}}\right)^{-1} .
$$

This shows that $f \in L \Gamma_{\alpha}\left(\mathrm{B}^{n}\right)$. The proof is complete.

Next, we observe that if $\Phi_{1}(t)=t^{p}$ or $\left(\frac{t}{\log (e+t)}\right)^{p}$ and $\Phi_{2}(t)=t^{q}$, $0<p, q<1$, then $\varrho_{1}(t)=t^{\frac{1}{p}-1}$ or $\frac{t^{\frac{1}{p}-1}}{\log \frac{e}{t}}$ respectively, and $\Psi_{\varrho_{2}}(t) \simeq 1$. 
Consequently, $\varrho:=\frac{\varrho_{1}}{\Psi_{\varrho_{2}}} \simeq \varrho_{1}$. It follows from this and the remark above that Theorem 1.2 can be interpreted in this case as follows.

Proposition 2.7. Let $0<p, q<1$ and $\alpha=n\left(\frac{1}{p}-1\right)$. Then the Hankel operator $h_{b}$ extends into a bounded operator

(i) from $\mathscr{H}^{p}\left(\mathrm{~B}^{n}\right)$ to $\mathscr{H}^{q}\left(\mathrm{~B}^{n}\right)$ if and only if $b \in \Gamma_{\alpha}\left(\mathrm{B}^{n}\right)$,

(ii) from $\mathscr{H}_{p}\left(\mathrm{~B}^{n}\right)$ to $\mathscr{H}^{q}\left(\mathrm{~B}^{n}\right)$ if and only if $b \in L \Gamma_{\alpha}\left(\mathrm{B}^{n}\right)$.

We will need the following lemma.

Lemma 2.8. For $\beta>0$ and $\gamma>0$, there exists a constant $C>0$ such that, for any $w \in \mathrm{B}^{n}$,

$$
I(w)=\int_{S^{n}}\left|\log \left(\frac{1-\langle\xi, w\rangle}{1-|w|^{2}}\right)\right|^{\gamma} \frac{\left(1-|w|^{2}\right)^{\beta}}{|1-\langle\xi, w\rangle|^{n+\beta}} d \sigma(\xi) \leq C .
$$

Proof. We follow the proof of Lemma 3.1 of [3]. It is easy to see that for $\xi \in \mathrm{S}^{n}$ and $w \in \mathrm{B}^{n}$,

$$
\left|\frac{1-\langle\xi, w\rangle}{1-|w|^{2}}\right| \geq \frac{1}{2}
$$

Now, from the fact that for $\varepsilon>0$, there exists a constant $C>0$ such that $\log ^{+} x \leq C x^{\varepsilon}$, we obtain that

$$
\left|\log \left(\frac{1-\langle\xi, w\rangle}{1-|w|^{2}}\right)\right| \leq C\left|\frac{1-\langle\xi, w\rangle}{1-|w|^{2}}\right|^{\varepsilon} .
$$

Taking $\varepsilon<\beta / \gamma$ and using Proposition 1.4.10 of [12], we obtain

$$
I(w) \leq C \int_{S^{n}} \frac{\left(1-|w|^{2}\right)^{\beta-\varepsilon \gamma}}{|1-\langle\xi, w\rangle|^{n+\beta-\varepsilon \gamma}} d \sigma(\xi) \leq C .
$$

\section{The boundedness of $h_{b}: \mathscr{H}^{p}\left(\mathrm{~B}^{n}\right) \rightarrow \mathscr{H}^{q}\left(\mathrm{~B}^{n}\right)$ for $0<p<q, q \geq 1$}

We start this section with the case $q=1$. We have the following result.

TheOREm 3.1. Let $0<p<1$ and $\alpha=n\left(\frac{1}{p}-1\right)$. Then the Hankel operator $h_{b}$ extends as a bounded operator from $\mathscr{H}^{p}\left(\mathrm{~B}^{n}\right)$ into $\mathscr{H}^{1}\left(\mathrm{~B}^{n}\right)$ if and only if $b \in L \Gamma_{\alpha}$. Moreover we have $\left\|h_{b}\right\| \simeq\|b\|_{L \Gamma_{\alpha}}$.

Proof. First, we suppose that the function $b \in L \Gamma_{\alpha}\left(\mathrm{B}^{n}\right)$. That is $b \in \mathscr{H}\left(\mathrm{B}^{n}\right)$ and satisfies, for some $k>\alpha$, the condition

$$
\left|R^{k} b(z)\right| \leq C\left(1-|z|^{2}\right)^{n\left(\frac{1}{p}-1\right)-k}\left(\log \frac{1}{1-|z|^{2}}\right)^{-1} .
$$


We prove that $h_{b}$ is bounded from $\mathscr{H}^{p}\left(\mathrm{~B}^{n}\right)$ into $\mathscr{H}^{1}\left(\mathrm{~B}^{n}\right)$. For this, we recall that

$$
\begin{aligned}
h_{b}(f)(z) & =P(b \bar{f})(z) \\
& =\int_{\mathrm{S}^{n}} b(\xi) \overline{f(\xi)} S(z, \xi) d \sigma(\xi) \\
& =C_{k} \int_{\mathrm{B}^{n}} S(z, w) M_{k} b(w) \overline{f(w)}\left(1-|w|^{2}\right)^{k-1} d v(w) .
\end{aligned}
$$

Now, by Proposition 1.4.10 of [12], we have

$$
\int_{S^{n}}|S(r \eta, w)| d \sigma(\eta) \approx \log \frac{1}{1-r|w|} .
$$

Combining these facts with the inequality (2.5), we obtain

$$
\begin{aligned}
& \int_{\mathrm{S}^{n}}\left|h_{b} f(r \eta)\right| d \sigma(\eta) \\
& \quad \leq C \int_{\mathrm{S}^{n}} \int_{\mathrm{B}^{n}}|S(r \eta, w)|\left|M_{k} b(w)\right||f(w)|\left(1-|w|^{2}\right)^{k-1} d \nu(w) d \sigma(\eta) \\
& \quad \leq C \int_{\mathrm{B}^{n}}\left|M_{k} b(w)\right|\left(\log \frac{1}{1-r|w|}\right)\left(1-|w|^{2}\right)^{k-1}|f(w)| d \nu(w) \\
& \leq C\|b\|_{L \Gamma_{\alpha}\left(\mathrm{B}^{n}\right)} \int_{\mathrm{B}^{n}}|f(w)|\left(1-|w|^{2}\right)^{n\left(\frac{1}{p}-1\right)-1} d \nu(w) \\
& \quad \leq C\|b\|_{L \Gamma_{\alpha}\left(\mathrm{B}^{n}\right)}\|f\|_{\mathscr{C}^{p}} .
\end{aligned}
$$

This complete the first part of the proof. Conversely, if $h_{b}$ is bounded from $\mathscr{H}^{p}\left(\mathrm{~B}^{n}\right)$ into $\mathscr{H}^{1}\left(\mathrm{~B}^{n}\right)$, then we have for every $f \in \mathscr{H}^{p}\left(\mathrm{~B}^{n}\right)$ and $g \in B M O A=$ $\left(\mathscr{H}^{1}\left(\mathrm{~B}^{n}\right)\right)^{*}$,

$$
\left|\left\langle h_{b}(f), g\right\rangle\right|=|\langle b, f g\rangle| \leq C\left\|h_{b}\right\|\|f\|_{\mathscr{H} p}\|g\|_{B M O A} .
$$

Let $w \in \mathrm{B}^{n}$, we will apply the inequality (3.1) to $f$ and $g$, with

$$
f(z)=f_{w}(z)=\frac{\left(1-|w|^{2}\right)^{k-n\left(\frac{1}{p}-1\right)}}{(1-\langle z, w\rangle)^{n+k}}
$$

and

$$
g(z)=\log (1-\langle z, w\rangle)
$$


where $k$ is an integer with $k>n\left(\frac{1}{p}-1\right)$. Since $g$ is uniformly in $B M O A$ and $f$ is also uniformly in $\mathscr{H}^{p}\left(\mathrm{~B}^{n}\right)$ by [12, Proposition 1.4.10], it follows that

$$
\begin{aligned}
\mid \lim _{r \rightarrow 1} \int_{S^{n}} \frac{b(\xi)}{(1-\langle w, r \xi\rangle)^{n+k}}\left(1-|w|^{2}\right)^{k-n\left(\frac{1}{p}-1\right)} & \log (1-\langle w, r \xi\rangle) d \sigma(\xi) \mid \leq C\left\|h_{b}\right\| .
\end{aligned}
$$

This is equivalent to

$$
\begin{aligned}
\mid \lim _{r \rightarrow 1} \int_{S^{n}} \frac{\left(1-|w|^{2}\right)^{k-n\left(\frac{1}{p}-1\right)} b(\xi)}{(1-\langle w, r \xi\rangle)^{n+k}} & \log \left(1-|w|^{2}\right) d \sigma(\xi) \\
& +\lim _{r \rightarrow 1} \int_{S^{n}} b(\xi) \overline{h(r \xi)} d \sigma(\xi) \mid \leq C\left\|h_{b}\right\|
\end{aligned}
$$

where

$$
h(z)=\frac{\left(1-|w|^{2}\right)^{k-n\left(\frac{1}{p}-1\right)}}{(1-\langle z, w\rangle)^{n+k}} \log \left(\frac{1-\langle z, w\rangle}{1-|w|^{2}}\right) .
$$

By Lemma $2.8, h$ is uniformly in $\mathscr{H}^{p}\left(\mathrm{~B}^{n}\right)$. That is $\|h\|_{\mathscr{H}^{p}\left(\mathrm{~B}^{n}\right)} \leq C$.

An application of (3.1) to $f=h$ and $g=1$ gives

$$
\left|\lim _{r \rightarrow 1} \int_{S^{n}} b(\xi) \overline{h(r \xi)} d \sigma(\xi)\right| \leq C\left\|h_{b}\right\| .
$$

So that

$$
\left|\lim _{r \rightarrow 1} \int_{\mathbf{S}^{n}} \frac{\left(1-|w|^{2}\right)^{k-n\left(\frac{1}{p}-1\right)} b(\xi)}{(1-\langle w, r \xi\rangle)^{n+k}} \log \left(1-|w|^{2}\right) d \sigma(\xi)\right| \leq C\left\|h_{b}\right\|
$$

or equivalently

$$
\begin{aligned}
&\left|\lim _{r \rightarrow 1} \int_{S^{n}} \frac{b(\xi)}{(1-\langle w, r \xi\rangle)^{n+k}} d \sigma(\xi)\right| \\
& \leq C\left\|h_{b}\right\|\left(1-|w|^{2}\right)^{n\left(\frac{1}{p}-1\right)-k}\left(\log \frac{1}{1-|w|^{2}}\right)^{-1} .
\end{aligned}
$$

That is, for all $w \in \mathrm{B}^{n}$,

$$
\left|M_{k} b(w)\right| \leq C\left\|h_{b}\right\|\left(1-|w|^{2}\right)^{n\left(\frac{1}{p}-1\right)-k}\left(\log \frac{1}{1-|w|^{2}}\right)^{-1} .
$$

The proof is complete. 
In the case $h_{b}: \mathscr{H}^{p}\left(\mathrm{~B}^{n}\right) \rightarrow \mathscr{H}^{q}\left(\mathrm{~B}^{n}\right)$ with $0<p<q<\infty$, and $q>1$, we have the following result.

Theorem 3.2. Let $q>1,0<p<q<\infty$ and $\alpha=n\left(\frac{1}{p}-\frac{1}{q}\right)$. Then the Hankel operator $h_{b}$ extends into a bounded operator from $\mathscr{H}^{p}\left(\mathrm{~B}^{n}\right)$ into $\mathscr{H}^{q}\left(\mathrm{~B}^{n}\right)$ if and only if its symbol b belongs to $\Gamma_{\alpha}\left(\mathrm{B}^{n}\right)$.

Moreover we have $\left\|h_{b}\right\| \simeq\|b\|_{\Gamma_{\alpha}\left(\mathrm{B}^{n}\right)}$.

Proof. We first prove the sufficiency of the condition for the boundedness. Let $\frac{1}{r}=\frac{1}{p}+\frac{1}{q^{\prime}}=1+\frac{1}{p}-\frac{1}{q}>1$, where $q^{\prime}$ is the conjugate exponent of $q$. It is not difficult to see that for $f \in \mathscr{H}^{p}\left(\mathrm{~B}^{n}\right)$ and $g \in \mathscr{H}^{q^{\prime}}\left(\mathrm{B}^{n}\right), f g \in \mathscr{H}^{r}\left(\mathrm{~B}^{n}\right)$ with $\|f g\|_{r} \leq\|f\|_{\mathscr{H}^{p}}\|g\|_{q^{\prime}}$. Since we know that $\left(\mathscr{H}^{r}\left(\mathrm{~B}^{n}\right)\right)^{*}=\Gamma_{\alpha}\left(\mathrm{B}^{n}\right)$ (with equivalent norms) with $\alpha=n\left(\frac{1}{r}-1\right)=n\left(\frac{1}{p}-\frac{1}{q}\right)$. It follows that there exists a positive constant $C$ such that

$$
\left|\left\langle h_{b}(f), g\right\rangle\right|=|\langle b, f g\rangle| \leq C\|b\|_{\Gamma_{\alpha}\left(\mathrm{B}^{n}\right)}\|f\|_{\mathscr{H} p}\|g\|_{q^{\prime}} .
$$

We conclude that if $b \in \Gamma_{\alpha}\left(\mathrm{B}^{n}\right)$, then $h_{b}$ is bounded from $\mathscr{H}^{p}\left(\mathrm{~B}^{n}\right)$ into $\mathscr{H}^{q}\left(\mathrm{~B}^{n}\right)$ with $\left\|h_{b}\right\| \leq C\|b\|_{\Gamma_{\alpha}\left(\mathrm{B}^{n}\right)}$.

Conversely, suppose that $h_{b}$ extends into a bounded operator from $\mathscr{H}^{p}\left(\mathrm{~B}^{n}\right)$ into $\mathscr{H}^{q}\left(\mathrm{~B}^{n}\right)$. Then as previously remarked,

$$
\left|\lim _{r \rightarrow 1} \int_{S^{n}} b(\xi) \overline{f(r \xi) g(r \xi)} d \sigma(\xi)\right| \leq C\left\|h_{b}\right\|\|f\|_{\mathscr{C}^{p}}\|g\|_{q^{\prime}} .
$$

Let $w \in \mathrm{B}^{n}$, we apply this inequality to $f(z)=f_{w}(z)=\frac{1}{(1-\langle z, w\rangle)^{k}}, g(z)=$ $\frac{1}{(1-\langle z, w\rangle)^{n}}$, with $k>\frac{n}{p}$. From Proposition 1.4.10 of [12], we get

$$
\|f\|_{\mathscr{H}^{p}} \leq C\left(1-|w|^{2}\right)^{\frac{n}{p}-k} ; \quad\|g\|_{q^{\prime}} \leq C\left(1-|w|^{2}\right)^{-\left(\frac{n}{q}\right)} .
$$

It follows that

$$
\left|\lim _{r \rightarrow 1} \int_{\mathbf{S}^{n}} \frac{b(\xi)}{(1-\langle w, r \xi\rangle)^{n+k}} d \sigma(\xi)\right| \leq C\left\|h_{b}\right\|\left(1-|w|^{2}\right)^{n\left(\frac{1}{p}-\frac{1}{q}\right)-k} .
$$

That is, for all $w \in \mathrm{B}^{n}$,

$$
\left|M_{k} b(w)\right| \leq C\left\|h_{b}\right\|\left(1-|w|^{2}\right)^{n\left(\frac{1}{p}-\frac{1}{q}\right)-k},
$$

So $\|b\|_{\Gamma_{\alpha}\left(\mathrm{B}^{n}\right)} \leq C\left\|h_{b}\right\|$. This completes the proof of the theorem. 


\section{The boundedness of $h_{b}: \mathscr{H}_{p}\left(\mathrm{~B}^{n}\right) \rightarrow \mathscr{H}^{q}\left(\mathrm{~B}^{n}\right)$ for $0<p \leq 1, q \geq 1$}

We have the following result in the case $q=1$.

THEOREM 4.1. Let $0<p<1$ and $\alpha=n\left(\frac{1}{p}-1\right)$. Then the Hankel operator $h_{b}$ extends as a bounded operator from $\mathscr{H}_{p}\left(\mathrm{~B}^{n}\right)$ into $\mathscr{H}^{1}\left(\mathrm{~B}^{n}\right)$ if and only if $b \in L \Gamma_{\alpha}^{2}\left(\mathrm{~B}^{n}\right)$. Moreover we have $\left\|h_{b}\right\| \simeq\|b\|_{L \Gamma_{\alpha}^{2}\left(\mathrm{~B}^{n}\right)}$.

Proof. The sufficiency part can be handled as in Theorem 3.1 with the help of the inequality (2.6). The proof of the necessity also follows the steps of the proof of Theorem 3.1. We sketch here the necessary changes.

If $h_{b}$ is bounded from $\mathscr{H}_{p}\left(\mathrm{~B}^{n}\right)$ into $\mathscr{H}^{1}\left(\mathrm{~B}^{n}\right)$, then we have for every $f \in$ $\mathscr{H}_{p}\left(\mathrm{~B}^{n}\right)$ and $g \in B M O A$,

$$
\left|\left\langle h_{b}(f), g\right\rangle\right|=|\langle b, f g\rangle| \leq C\left\|h_{b}\right\|\|f\|_{\mathscr{H}_{p}}\|g\|_{B M O A} .
$$

This inequality will play the role of the inequality (3.1). Apply it to $f=m l$ and $g$, with

$$
m(z)=m_{w}(z)=\frac{\left(1-|w|^{2}\right)^{k-n\left(\frac{1}{p}-1\right)}}{(1-\langle z, w\rangle)^{n+k}} \quad \text { and } \quad l(z)=g(z)=\log (1-\langle z, w\rangle)
$$

where $k$ is an integer with $k>n\left(\frac{1}{p}-1\right)$, to obtain using Theorem 1.1, that

$$
\begin{aligned}
\lim _{r \rightarrow 1} \int_{S^{n}} \frac{b(\xi)}{(1-\langle w, r \xi\rangle)^{n+k}}\left(1-|w|^{2}\right)^{k-n\left(\frac{1}{p}-1\right)} & (\log (1-\langle w, r \xi\rangle))^{2} d \sigma(\xi) \mid \leq C\left\|h_{b}\right\| .
\end{aligned}
$$

Now, observing, by direct computation, that

$$
\begin{aligned}
\log ^{2}(1-\langle w, r \xi\rangle)=\log ^{2}\left(1-|w|^{2}\right)-\log ^{2}\left(\frac{1-\langle w, r \xi\rangle}{1-|w|^{2}}\right) \\
+2 \log (1-\langle w, r \xi\rangle) \log \left(\frac{1-\langle w, r \xi\rangle}{1-|w|^{2}}\right),
\end{aligned}
$$

we proceed as in the end of the proof of Theorem 3.1, with the natural changes, to show that, for $w \in \mathrm{B}^{n}$,

$$
\left|M_{k} b(w)\right| \leq C\left\|h_{b}\right\|\left(1-|w|^{2}\right)^{n\left(\frac{1}{p}-1\right)-k}\left(\log \frac{1}{1-|w|^{2}}\right)^{-2} .
$$

The proof is complete. 
In the case $h_{b}: \mathscr{H}_{p}\left(\mathrm{~B}^{n}\right) \rightarrow \mathscr{H}^{q}\left(\mathrm{~B}^{n}\right)$ for $q>1$ and $0<p \leq 1$, we have the following result.

Theorem 4.2. Let $q>1,0<p \leq 1$ and $\alpha=n\left(\frac{1}{p}-\frac{1}{q}\right)$. The Hankel operator $h_{b}$ extends into a bounded operator from $\mathscr{H}_{p}\left(\mathrm{~B}^{n}\right)$ into $\mathscr{H}^{q}\left(\mathrm{~B}^{n}\right)$ if and only if its symbol b belongs to $L \Gamma_{\alpha}\left(\mathrm{B}^{n}\right)$.

Moreover $\left\|h_{b}\right\| \simeq\|b\|_{L \Gamma_{\alpha}\left(\mathrm{B}^{n}\right)}$.

PROOF. The sufficient condition is proved as in Theorem 3.2. We provide with details here. Let $\frac{1}{r}=\frac{1}{p}+\frac{1}{q^{\prime}}=1+\frac{1}{p}-\frac{1}{q}>1$. It is enough to prove that for $f \in \mathscr{H}_{p}\left(\mathrm{~B}^{n}\right)$ and $g \in \mathscr{H}^{q^{\prime}}\left(\mathrm{B}^{n}\right), f g \in \mathscr{H}_{r}\left(\mathrm{~B}^{n}\right)$ with

$$
\|f g\|_{\mathscr{H}_{r}} \lesssim\|f\|_{\mathscr{H}_{p}}\|g\|_{q^{\prime}} .
$$

Indeed, if the latter holds, then since $\left(\mathscr{H}_{r}\left(\mathrm{~B}^{n}\right)\right)^{*}=L \Gamma_{\alpha}\left(\mathrm{B}^{n}\right)$ (with equivalent norms) with $\alpha=n\left(\frac{1}{r}-1\right)=n\left(\frac{1}{p}-\frac{1}{q}\right)$, it would follow that there exists a positive constant $C$ such that

$$
\left|\left\langle h_{b} f, g\right\rangle\right|=|\langle b, f g\rangle| \leq C\|b\|_{L \Gamma_{\alpha}\left(\mathrm{B}^{n}\right)}\|f\|_{\mathscr{P}_{p}}\|g\|_{q^{\prime}} .
$$

This gives that if $b \in L \Gamma_{\alpha}\left(\mathrm{B}^{n}\right)$, then $h_{b}$ is bounded from $\mathscr{H}_{p}\left(\mathrm{~B}^{n}\right)$ into $\mathscr{H}^{q}\left(\mathrm{~B}^{n}\right)$ with $\left\|h_{b}\right\| \leq C\|b\|_{L \Gamma_{\alpha}\left(\mathrm{B}^{n}\right)}$.

Let us prove (4.4). We recall that $\Phi_{r}(t)=\left(\frac{t}{\log (e+t)}\right)^{r}$ is nondecreasing. Thus, for $a>0$ and $b>0$, we have $\Phi_{r}(a b) \leq \Phi_{r}(b)$ if $a \leq 1$, and for $a>1$ the obvious estimate $\log (e+a b) \geq \log (e+b)$ gives $\Phi_{r}(a b) \leq a^{r} \Phi_{r}(b)$. It follows that if $\epsilon>0$ and $C>0$ are the constants in (1.1), then taking $a=\frac{|g|}{\|g\|_{q^{\prime}}}$ and $b=\frac{|f|}{(2 C)^{1 / \epsilon} \lambda}, \lambda>0, f \in \mathscr{H}_{p}\left(\mathrm{~B}^{n}\right)$, and applying Hölder's inequality, we obtain

$$
\int_{S^{n}} \Phi_{r}\left(\frac{|f(\xi)||g(\xi)|}{\lambda(2 C)^{1 / \epsilon}\|g\|_{q^{\prime}}}\right) d \sigma(\xi) \leq\left(\int_{S^{n}} \Phi_{p}\left(\frac{|f(\xi)|}{\lambda}\right) d \sigma(\xi)\right)^{r / p} .
$$

It follows that if $\lambda$ is any positive number such that

$$
\int_{S^{n}} \Phi_{p}\left(\frac{|f(\xi)|}{\lambda}\right) d \sigma(\xi) \leq 1,
$$

then $\|f g\|_{L^{\Phi_{r}}}^{\operatorname{lux}} \leq(2 C)^{1 / \epsilon} \lambda\|g\|_{q^{\prime}}$. Therefore, $\|f g\|_{L^{\Phi_{r}}}^{\operatorname{lux}} \leq(2 C)^{1 / \epsilon}\|f\|_{L^{\Phi_{p}}}^{\operatorname{lux}}\|g\|_{q^{\prime}}$. We conclude that for $f \in \mathscr{H}_{p}\left(\mathrm{~B}^{n}\right)$ and $g \in \mathscr{H}^{q^{\prime}}\left(\mathrm{B}^{n}\right)$,

$$
\|f g\|_{\mathscr{H}_{r}} \lesssim\|f\|_{\mathscr{H}_{p}}\|g\|_{q^{\prime}}
$$


Next, we suppose that $h_{b}$ extends into a bounded operator from $\mathscr{H}_{p}\left(\mathrm{~B}^{n}\right)$ into $\mathscr{H}^{q}\left(\mathrm{~B}^{n}\right)$. Then,

$$
\left|\lim _{r \rightarrow 1} \int_{S^{n}} b(\xi) \overline{f(r \xi) g(r \xi)} d \sigma(\xi)\right| \leq C\left\|h_{b}\right\|\|f\|_{\mathscr{H}_{p}}\|g\|_{q^{\prime}} .
$$

We apply this inequality to $f=m l$ with $m(z)=m_{w}(z)=\frac{1}{(1-\langle z, w\rangle)^{k}}$, $l(z)=\log (1-\langle z, w\rangle)$, and $g(z)=\frac{1}{(1-\langle z, w\rangle)^{n}}$, with $k>\frac{n}{p}$, where $w \in \mathrm{B}^{n}$ is fixed. The rest of the proof follows as in the necessity part of Theorem 3.2. We omit the details. The proof is complete.

ACKNOWLEDGEMENTS. This work was done while the first author was a research fellow at the School of Mathematics of Trinity College of Dublin and the second author was a post-doctoral fellow at the Centre of Recerca Matemàtica, Barcelona (Spain). The former was supported by the "Irish Research Council for Science, Engineering and Technology" and the latter by the Centre of Recerca Matemàtica, Barcelona (Spain).

\section{REFERENCES}

1. Bonami, A., and Grellier, S., Hankel operators and weak factorization for Hardy-Orlicz spaces, Colloq. Math. 118 (2010), 107-132.

2. Bonami, A., Grellier, S., and Sehba, B. F., Boundedness of Hankel operators on $\mathscr{H}^{1}\left(\mathrm{~B}^{n}\right)$, C. R. Math. Acad. Sci. Paris 344 (2007), 749-752.

3. Bonami, A., and Luo, L., On Hankel operators between Bergman spaces on the unit ball, Houston J. Math. 31 (2005), 815-827.

4. Bonami, A., and Madan, S., Balayage of Carleson measures and Hankel operators on generalized Hardy spaces, Math. Nachr. 153 (1991), 237-245.

5. Bonami, A., Peloso, M. M., and Symesak, F., Factorization of Hardy spaces and Hankel operators on convex domains in $\mathrm{C}^{n}$, J. Geom. Anal. 11 (2001), 363-397.

6. Bonami, A., and Sehba, B. F., Hankel operators between Hardy-Orlicz spaces and products of holomorphic functions, Rev. Math. Arg. 50 (2009), 187-199.

7. Coifman, R., Rochberg, R., and Weiss, G., Factorization theorems for Hardy spaces in several variables, Ann. of Math. (2) 103 (1976), 611-635.

8. Janson, S., On functions with conditions on the mean oscillation, Ark. Mat. 14 (1976), 189196.

9. Janson, S., Generalizations of Lipschitz spaces and an application to Hardy spaces and bounded mean oscillation, Duke Math. J. 47 (1980), 959-982.

10. Janson, S., Peetre, J., and Semmes, S., On the action of Hankel and Toeplitz operators on some function spaces, Duke Math. J. 51 (1984), 937-958.

11. Mesrar, Y., Inégalités $H^{p}$ pour les opérateurs de Hankel a symbole lipschitzien, these, l'Université d'Orléans U. F. R. Faculté des sciences 1987.

12. Rudin, W., Function theory in the unit ball of $\mathrm{C}^{n}$, Grundlehren Math. Wiss. 241, Springer, Berlin 1980.

13. Sehba, B. F., On some equivalent definitions of $\rho$-Carleson measures, Acta Sci. Math. (Szeged) 75 (2009), 499-525.

14. Smith, W. S., BMO ( $\rho)$ and Carleson measures, Trans. Amer. Math. Soc. 287 (1985), 107-126. 
15. Tolokonnikov, V. A., Hankel and Toeplitz operators in Hardy spaces, J. Sov. Math. 37 (1987), 1359-1364.

16. Zhu, K., Spaces of holomorphic functions in the unit ball, Grad. Texts Math. 226, Springer, Berlin 2005.

CENTRE DE MATHÉMATIQUES ET INFORMATIQUE (CMI) UNIVERSITÉ DE PROVENCE

TECHNOPÔLE CHÂTEAU-GOMBERT

39, RUE F. JOLIOT CURIE

13453 MARSEILLE CEDEX 13

FRANCE

E-mail: bsehba@cmi.univ-mrs.fr
DÉPARTEMENT DE MATHÉMATIQUES

FACULTÉ DES SCIENCES

DE L'UNIVERSITÉ DE YAOUNDÉ I

B.P. 812 YAOUNDÉ

CAMEROUN

E-mail: tchoundjaedgar@yahoo.fr 